\title{
Microbial diversity in raw milk and Sayram Ketteki from southern of Xinjiang, China
}

DongLa Gao ${ }^{1,2}$, Weihua Wang ${ }^{1,2 *}$, ZhanJiang Han ${ }^{1,3}$, Qian $\mathrm{Xi}^{1,2}$, , RuiCheng Guo ${ }^{1,2}$, PengCheng Kuang ${ }^{1,2}$, DongLiang $\mathrm{Li}^{1,2}$

1 College of Life Science, Tarim University, Alaer, Xinjiang, China

2 Xinjiang Production and Construction Corps Key Laboratory of Deep Processing of Agricultural Products in South Xinjiang, Alar, Xinjiang, China

3 Xinjiang Production and Construction Corps Key Laboratory of Protection and Utilization of Biological Resources in Tarim Basin, Alar, Xinjiang, China

*Corresponding author

E-mail: wangweihua6688@163.com (Weihua Wang) 


\section{Abstract}

Raw milk and fermented milk are rich in microbial resources, which are essential for the formation of texture, flavor and taste. In order to gain a deeper knowledge of the bacterial and fungal community diversity in local raw milk and home-made yogurts from Sayram town, Baicheng county, Akesu area, southern of Xinjiang, China,30 raw milk and 30 home-made yogurt samples were collected and experiment of high-throughput sequencing was implemented.The results of experiments revealed the species of fungi in raw milk was the most, and the species of bacteria in fermented milk was the least.Based on principal component analysis (PCA), it was found that the bacterial and fungal community structure differed in samples from two types of dairy products. And the presence of 15 bacterial and 12 fungal phyla, comprising 218 bacterial and 495 fungal genera respectively, among all samples. Firmicutes and Ascomycota,Lactobacillus and Candida were the predominant phyla and genera of bacteria and fungi, respectively. The results indicated that the microbial community of raw milk differs from home-made yogurts due to sampling location and manufacturing process. The study suggested that high-throughput sequencing could provide a better understanding of microbiological diversity as well as lay a theoretical foundation for selecting beneficial microbial resources from this natural yogurt.

Keywords: high-throughput sequencing; microbial diversity; raw milk; Sayram Ketteki

\section{Introduction}

Raw milk (RM) and dairy products derived therefrom are rich in nutrients and microorganisms, and their microbial composition have been discovered in previous studies, through traditional culture-dependent and culture-independent methods [1,2].Microbial 
differences in RM are related to many factors, such as animal sources and geographic locations. In the flora of 14 pastures, the leading bacteria genera were Enterococcus, Bacillus, Acinetobacter and Lactococcus but only Staphylococcus aureus and Shigella were detected in a few cattle farms [3]. In donkey milk, it was found that the dominant bacterial phyla were Proteobacteria and Firmicutes with dominant genera being Pseudomonas, Ralstonia and Acinetobacter[4]. In goat milk, it was found that the dominant genera were proteobacteria and Enterobacter among 354 genera[5].

Generally, the most dominant bacterial genera in dairy products are lactobacillus, lactococcus, streptococcus and leuconostoc of Firmicutes, and they affect the flavor and maturity of fermentation dairy products [6,7]. For example, lactic acid bacteria play an important role in the fermentation process, in which they heterogeneously ferment to generate ethanol, carbon dioxide, acetaldehyde, acetone, acetoin, diacetyl and other volatile aromatic compounds, promoting the formation of good food flavors[8].Yeast fermented dairy products, such as kefir, koumiss and mozzarella, are typical fermented milk with alcohols, which are important contributors to fragrance and wine aroma [9]. It was revealed that Geotrichum candidum was a main contributor to the maturation of camemberti cheese and had the ability to metabolize amino bitter peptides and enhance the sulfur taste[10] .

Although traditional cultivation methods and molecular technologies made us have a preliminary understanding of microbial composition in dairy products [11,12], the information obtained is not always accurate and the details of these products' microbial diversity remains elusive. The main reason is that a large number of microbial species could not be cultured successfully [13].

In the recent years, with the mutual penetration of genetics, bioinformatics and other disciplines, more and more culture-free technologies have been used in the studies of microbial diversity[14]. Metagenomics[15] is a fast and efficient method which uses 
high-throughput sequencing to simultaneously sequence millions of DNA molecules, and constructs a metagenome library by detecting the genetic material sequences of variable regions in microorganisms, exploring the diversity of microbial species and community structure. This technology avoids non-cultivable and low-abundance microbial unpredictability, and objectively restores the community structure and abundance ratio, maximizing the development of microbial resources. This approach has been widely used in medical and environmental science [16, 17], and gradually used in the food science[18].

Through metagenomics, the diversity of microorganisms, dominant flora and metabolic potential in Mexican Cotija cheese was discovered[19]. It indicated that the Lactobacillus, Leuconostoc and Weissella were the main dominant flora, the microbial metabolic activities related to the formation of multiple flavors mainly were involved the metabolism of branched chain amino acids and free fatty acids, and also discovered some genes related to bacteriocin production and immunity. Shewanella, Acinetobacter, Pelomonas, Dysgonomonas, Weissella and Pseudomonas were found in Tibetan kefir grains for the first time[20]. In Turkish Kefir grains, it was found that communities in the two samples showed high consistency. Lactobacillus including L. kefiranofaciens, L. buchneri and L. helveticus was the most abundant genus[21]. The diversity of fungi in French Tomme d'Orchies cheese was interesting. The results showed that it contained Yarrowia lipolytica and Galactomyces geotrichum, and some rare microorganisms had been discovered, such as Clavis-poralusitaniae, Kazachstania unispora and Cladosporium cladosporioides[22].

The microbial diversity of fermented dairy products, such as koumiss[23], jueke [24], tarag [25] and airag [26], had been investigated widely, but there were few reports on fermented dairy products in Xinjiang province of China [27], especially in south Xinjiang. Sayram Ketteki (SK), a traditional and popular handcraft fermented food, was produced by local Uighur peasant woman in Xinjiang province of China. To generate SK, the milk from 
cows is used as the raw material, and a little yogurt reserved in the previous batch acts as starter of fermentation. Because of continuous making yogurt, the fermentation starters from some families even have decades of history. After fermenting in porcelain bowels at $10-25^{\circ} \mathrm{C}$ about 6-8 hours, it obtains the products with the color of milky white, elastic, sweet and sour-tasting with a slightly alcoholic. SK was typical alcoholic fermented milk. Several studies have investigated the lactic acid bacteria and yeasts in SK using culture-based method and molecular biological identification. It was reported that the dominant strains of SK were L.Bulgaricus, Streptococcus thermophilus and Saccharomyces cerevisiae[28]. But some others studies demonstrated that L. helveticus, Streptococcus thermophilus and Kluyveromycus marxianus were the predominant [29]. Although there are some related studies[30], the mechanism of its flavor formation is still unclear. Undoubtedly, the formation of flavor has a lot to do with microorganisms, therefore, unraveling microbial community diversity of SK and its raw materials is very necessary.

In the current study, to explore the microbial species diversity and the microbial composition in RM and SK, 30 RM and 30 home-made yogurt samples were collected, metagenomic sequencing were carried out. Through bioinformatics analyses, it was found that microorganisms in both types of dairy products were various. Here we report the results.

\section{Materials and Methods}

\subsection{Sample collection and DNA extraction}

Sixty samples were collected aseptically from different national minority families of Sayram town Baicheng county which located in the southern of Xinjiang (approx $41^{\circ} 48^{\prime} \mathrm{N}$, $\left.81^{\circ} 87^{\prime} \mathrm{E}\right)$ of China, where the average temperature was $27^{\circ} \mathrm{C}$ in late July 2019 . RM samples were named with the letter " $\mathrm{X}$ " $(\mathrm{X} 1, \mathrm{X} 2 \ldots . . \mathrm{X} 34)$, while SK samples were named with the letter "S" (S1, S2....S34), respectively. Each RM sample and each SK sample was corresponding, 
because they came from the same family. These collected samples were stored at $-20^{\circ} \mathrm{C}$ for further use.

Every sample was centrifuged at $10000 \mathrm{rpm}$ for $10 \mathrm{~min}$. The supernatant was discarded and the precipitate was left. Total microbial DNAs were extracted from 500 mg sediment for each sample using the DNeasy Power Soil Kit (QIAGEN, Netherlands), according to instructions of the manufacturer, and kept at $-20^{\circ} \mathrm{C}$ prior to future analysis. The quantity and quality of extracted DNAs were measured by NanoDrop ND-1000 spectrophotometer and $0.8 \%$ agarose gel electrophoresis.

\subsection{PCR amplification of 16SrDNA and ITS sequences}

The bacterial V3-V4 of 16SrRNA gene was amplified, the used primers were as follows: 338F: 5'-ACT CCT ACG GGA GGC AGCA-3' (forward) and 806R: 5'-GGA CTA CHV GGG TWT CTA AT-3' (reverse). For fungal community analysis, the internal transcribed spacer (ITS) gene was amplified using the primers: ITS5F: 5'-GGA AGT AAA AGT CGT AAC AAG G-3' (forward) and ITS1R: 5'-GCT GCG TTC TTC ATC GAT GC-3' (reverse). In the PCR experiments, the thermal cycling consisted of an initial denaturation at $98^{\circ} \mathrm{C}$ for $5 \mathrm{~min}, 25$ cycles of denaturation at $98^{\circ} \mathrm{C}$ for $30 \mathrm{~s}$, annealing at $52^{\circ} \mathrm{C}$ for $30 \mathrm{~s}$, and extension at $72{ }^{\circ} \mathrm{C}$ for 1 min, followed by a final extension at $72{ }^{\circ} \mathrm{C}$ for $5 \mathrm{~min}$.

PCR products were measured with $2 \%$ agarose gel electrophoresis, and the target fragments were collected by using gel recovery kit (AXYGEN, Dalian, China). Referring to the preliminary quantitative results of electrophoresis, the obtained_products were quantified with fluorescence and according to the quantitative results and sequencing volume requirements of each sample. Subsequently, each sample was mixed in corresponding proportion and paired-end $2 \times 300 \mathrm{bp}$ sequencing was _carried out using the Illumina MiSeq 
platform with MiSeq Reagent Kit v3 by Shanghai Personal Biotechnology Co., Ltd (Shanghai, China).

\subsection{Sequence quality control}

In order to fulfill the requirements of subsequent analyses, the all readings were screened and filtered using Quantitative Insights into Microbial Ecology (QIIME) (V1.8.0) software [31]. Briefly, the raw reads with exact matches to the barcodes were assigned to respective samples and identified as valid sequences. The low-quality sequences that were less than $150 \mathrm{bp}$, average Phred scores of less than 20, ambiguous, and contained mononucleotide repeats more than 8 bp were discarded. Paired-end reads were assembled using FLASH[32]. After chimera detection, the remaining high quality sequences were clustered into operational taxonomic units (OTUs) at 97\% sequence identity with UCLUST[33]. The highest sequence was taken as the representative sequence of the OTU. OTU taxonomic classification was conducted by BLAST searching and aligning the Prokaryotic sequences with the Greengenes database[34], and Eukaryotic sequences with the Unite database[35].According to the number of sequences included in each sample of each OTU, a matrix file of the abundance of OTU in each sample was constructed.

\subsection{Bioinformatics and statistical analysis}

Sequence data analyses were mainly conducted using QIIME and R packages (V3.2.0). OTU-level alpha diversity indices, such as Chao1 and ACE richness index, Shannon and Simpson diversity index, were calculated using the OTU table in QIIME. OTU-level ranked abundance curves were generated to compare the richness and evenness of OTUs among samples. Beta diversity analysis was performed to investigate the structural variation of 
microbial communities across samples visualized via Principal component analysis (PCA) .

The taxonomy compositions and abundances were visualized using GraPhlAn [36], and correlations with $|\mathrm{RHO}|>0.6$ and $\mathrm{P}<0.01$ were visualized as co-occurrence network using Cytoscape. Microbial functions were predicted by PICRUSt (Phylogenetic investigation of communities by reconstruction of unobserved states) [37].

\section{Results}

\subsection{Bacterial and fungal sequence richness and diversity}

After filtering, we obtained 4,092,576 high-quality reads for bacteria, ranging from 60,078 to 75,419 with an average of $68,210 \pm 4,393$ sequence reads per sample. Meanwhile, 4,056,820 of ITS sequence reads were generated for the fungal community, ranging from 60,253 to 75,925 with an average of $67,614 \pm 4,665$ sequence reads per sample. The number of unique and classifiable representative OTUs sequences for bacteria and fungi were 2,221 and 4,062, respectively.

The 16SrRNA of bacteria from SK had the largest number of sequences, followed by the number of ITS sequences of fungi from RM and 16S_rRNA of bacteria from RM and the number of ITS sequences of fungi from SK was the smallest (Table 1). From the Chao1 index, the highest abundance of bacteria was in RM samples, followed by bacteria of SK, fungi of RM, and the lowest abundance was fungi of SK. From the Shannon index, the highest diversity was fungi of RM, followed by bacteria of RM and fungi of SK, and the bacteria of SK had the lowest diversity. The results of the numbers of OTUS were consistent with the results of diversity, which indicated that the species of fungi in RM was the most, and the species of bacteria in SK was the least.

The rarefaction curves, which can predict the total number of species and relative abundance of each species in a sample at a given sequencing depth indicated that when the 
prokaryotic sequencing volume was 40,000 , the bacterial sequence of each sample had not entered the plateau period (Fig 1A), and Shannon index curve (Fig 1B) indicated that all samples were still sequencing. When the amount reached 10,000, all OTUs were saturated. Although continued sequencing might discover new phylotypes, at this level, the bacterial diversity in the sample had already been captured. All the eukaryotic sequences at 10,000 had entered a gentle state, indicating that the sequencing volume of this experiment can truly reflect the diversity of fungi in the samples (Fig 1C).

Table 1: Alpha diversity values in samples

Fig 1.Bacterial rarefaction (A), Shannon diversity (B) and fungal rarefaction (C ) curves of populations of sixty samples. Different colored lines represent different samples.

\subsection{Comparison of bacterial and fungal community structure between RM and SK}

The contribution rates of the first principal component of the sample bacteria and fungi were $61.86 \%$ and $37.29 \%$, respectively, and the contribution rates of the second principal component were $10.4 \%$ and $21.3 \%$ (Fig 2). The closer the distance between the samples in the PCA diagram indicated the closer the composition of the two microbial communities. The SK and RM samples were separated on both sides, demonstrating that there was a significant difference in the bacterial group structure between the SK and the RM. The SK samples were almost stacked in a straight line, indicating that these samples had a high similar bacterial flora (Fig 2A). Simultaneously, the scattered distribution of bacterial communities in RM samples revealed that there were large differences between samples. The fungal samples (Fig 2B) showed a large aggregation and small dispersion. Most RM and SK samples were highly aggregated and overlapped, suggesting that these samples might have the similar fungal 
community, and a small part of the SK samples be scattered. This indicated that there was a difference in the fungal community among these samples.

Fig 2. Principal component analysis (PCA) of bacterial (A) and fungal (B) community. Red circle and green triangle represent samples of Sayram Ketteki and raw milk, respectively.

\subsection{Bacterial and fungal composition of RM and SK}

A total of 15 bacteria phyla were detected in 60 samples. Firmicutes (68.50\%), Proteobacteria (29.50\%), and Deinococcus-Thermus (1.10\%) were the dominant phyla, accounting for $99.1 \%$ of the total OTUs, while the Bacteroidetes, Actinobacteria, Cyanobacteria, Planctomycetes, Fusobacteria, Acidobacteria, Patescibacteria, Armatimonadetes, Verrucomicrobia, Elusimicrobia and Epsilonbacteraeota were observed simultaneously, indicating that the contents of them were very low, only accounting for $0.001 \%-0.006 \%$ of the total (Fig 3A).

A total of 218 genera of bacteria were detected, and there were 11 genera with abundance greater than 1\%, including Lactobacillus (45.30\%), Streptococcus (8.00\%), Macrococcus (7.70\%), Acinetobacter (6.70\%), Pseudomonas (6.00\%), Lactococcus (5.60\%), Enterobacter (4.50\%), Klebsiella (1.60\%), Ochrobactrum (1.40\%), Brevundimona (1.10\%) and Ralstonia (1.00\%) (Fig 5B). Moreover, it revealed that the Macrococcus, Acinetobacter, Pseudomonas and Lactococcus were the dominant genera in RM, with proportion being $15.3 \%, 13.1 \%, 11.9 \%$ and $11.15 \%$, respectively. Lactobacillus and Streptococcus in SK were the dominant bacterial genera, accounting for 98.1\% (Table 2 and Fig 3C). The content of Lactobacillus in these samples ranged from $36.4 \%$ to $99.7 \%$, which might be closely related to the sampling location, processing environment and other factors. The OTUs content of Lactobacillus and Streptococcus increased significantly after processing, while other 18 bacterial species decreased dramatically. For example, the OTUs content of Staphylococcus dropped from $1.66 \%$ to 0 , presuming that the staphylococcus was completely killed after heating treatment. 
A total of 12 fungal phyla were detected successfully (Fig 4A). Ascomycota (79.90\%), Basidiomycota (10.20\%) and Mucoromycota(3.40\%), with an average abundance value greater than $1 \%$ were absolutely dominant, accounting for $93.5 \%$ of the total, while the Mortierellomycota, Rozellomycota, Olpidiomycota, Entomophthoromycota, Chytridiomycota, Glomeromycota, Zoopagomycota, GS19 and other unidentified phylum, had very small content, only accounting for $0.1 \%-0.5 \%$ of the total OTUs. There were 495 fungal genera detected, and there were 18 genera with an average abundance value greater than $1 \%$, including Candida (21.20\%), Kluyveromyces (7.60\%), Aspergillus (5.10\%), Lecanicillium (3.90\%), Mucor $\quad(2.90 \%)$, Penicillium_(1.90\%) Clavispora (1.90\%) Malassezia (1.80\%), Yarrowia $(1.80 \%)$, Cladosporium (1.70\%), Thermoascus $\quad(1.70 \%)$, Simplicillium (1.50\%), Verticillium $(1.30 \%)$, Suillus (1.20\%), Pichia (1.20\%), Cutaneotrichosporon (1.20\%), Trichosporon (1.00\%) and Rhodotorula (1.00\%) (Fig 4B). It indicated that Candida had the highest content in the two types of samples (Table 2 and Fig 4C). After fermentation, the genera contents of Candida and Kluyveromyces increased significantly. The OTUS contents of Mucor, Clavispora, Yarrowia and Pichia increased slightly, and other 14 fungal genera decreased significantly.

In all, we found that the OTUs of predominant bacterial genera in SK was higher and obvious, and the OTUs of fungal genera were relatively uniform and small in both two types of dairy products (Table 2 ).

Fig 3. Relative abundances of bacteria at phylum (A) and genera (B) levels in each samples and GraPhlAn hierarchical tree map (C).

Fig 4. Relative abundances of fungi at phylum (A) and genera (B) levels in each samples and GraPhlAn hierarchical tree map (C). 
Table 2. Analysis of OTU Percentage of the top 20 genera

\subsection{Spearman association network analysis of dominant genera interaction}

The association network of dominant bacteria was constructed by using Cytoscape, and the most of the bacterial relationships were positive (Fig 5). The_Lactococcus was positively correlated with more than 20 species of bacteria for example Klebsiella, Staphylococcus, Enterobacter and so on, while the Lactobacillus was negatively correlated with more than 20 species of bacteria such as Enterococcus, Lactococcus and Streptococcus. It suggested that the lactic acid bacteria might ferment lactose to produce lactic acid, which created an acidic environment. The acid tolerance of Lactobacillus was higher than other bacteria, especially Lactococcus. This also explained the high abundance of Lactobacillus in yogurt, and higher abundance of Lactococcus in RM.

Fig 5. Dominant of top 50 bacterial genera association network diagram. The red and green lines indicates the positive and negative relationships, respectively.

\subsection{Prediction of flora metabolism function}

Using the PICRUSt, the prediction of bacterial metabolic functions can be achieved by comparing the existing 16SrRNA gene sequencing data with the microbial reference genome database with known metabolic functions. The composition of the KEGG two-level functional prediction information obtained from RM and SK was basically similar, but the abundance was obviously different (Fig 6A).

Bacterial community with higher abundance of RM was involved in the two classifications of carbohydrate metabolism and amino acid metabolism. SK had the largest variety of flora for carbohydrate metabolism. Venn diagram of common functional groups 
showed that although there were obvious differences in the composition of the microbial community, the functional groups in each type of sample were basically similar (Fig 6B).

Fig 6. PICRUSt predicted KEGG second level distribution map (A) and Venn diagram of common functional groups (B).

\section{Discussion}

The multiple microorganism and species diversity exist in RM[38] and traditional fermented dairy products[39, 40], and they play a crucial role in the formation of flavor. Studies have shown that the main source of the flavor substance acetaldehyde in yogurt is the synthesis of threonine by threonine aldolase secreted by the Bulgaria subspecies, and Streptococcus thermophilus can metabolize citric acid to produce diacetyl during yogurt fermentation and storage [41, 42]. There are various and popular fermentation dairy products in Xinjiang rural area, such as Kurut, Shubat, hence, there is no doubt that their particular flavor has a lot to do with the microbial polymorphism. However, the microbial diversity of these dairy products remain elusive. SK is one of the beloved yogurt and its microbial diversity has been not investigated yet, so, it's the first time to study its microbial polymorphism_in the current study.

Previous studies revealed that Lactobacillus is the main flora in cow's milk [43, 44]. In the current study, the Macrococcus, Acinetobacter, Pseudomonas and Lactococcus were the dominant bacterial genera in $\mathrm{RM}$, accounting for $51.5 \%$ of the total sequences, but the proportion of each sample is different. The Lactobacillus and Streptococcus were the dominant bacterial genera in SK, which are the similar to other studies [45]. At the fungal level, Candida was the absolutely advantageous genus in both two types of dairy products, while Aspergillus and 
Kluyveromyces were the subsequent dominant genera in RM and SK samples, which differed with others study [46]. The Kluyveromyces marxianus used in fermented food had the characteristics of weak alcohol and good aroma production, and could increase the content of free fatty acids and flavor substances in yogurt $[47,48]$. Maybe, that's why the flavor of SK is different from the other yogurts.

There were huge differences in the types and contents of microbial flora between samples. The most SK samples contained more than 50\% Lactobacillus and about $10 \%$ Streptococcus, but in S12, the contents of Lactobacillus and Streptococcus were $36.4 \%$ and $62.9 \%$, respectively. At fugal level, Candida was the dominant genus which the average content in RM was $13.4 \%$. In sample X12, Candida accounted for only $0.5 \%$, while Trichosporon accounted for $51.5 \%$. That may be caused by sampling. In the future, samples should be collected on a larger scale for further research.

In all, our findings partially explore the microbial diversity of RM and SK in south Xinjiang and contribute to providing a basis for industrialization SK. Besides, the microbial functional prediction can be helpful to search for new probiotics.

\section{Acknowledgments}

We would like to thank Dr Leilei Zhan for their helpful discussions and critical reading of the manuscript. 


\section{References}

1. Guo LD, Wang X, Du P, Zhao R, Huo GC. Study on Characteristics of Lactic Acid Bacteria Isolated from Traditional Indigenous Dairy Products. Food Ence, 2006. 27(3): 60-64.

2. Bao QH, Liu WJ, Yu J,Wang WH, Qing MJ, Chen X, et al.Isolation and identification of cultivable lactic acid bacteria in traditional yak milk products of Gansu Province in China. Journal of General \& Applied Microbiology, 2012.58(2): 95-105.

3. Yu GP, Chen Y,YaoYX, Fan MJ, Liu P, Dong LW. Application of Illumina MiSeq High-Throughput Sequencing Technique to Analyze Bacterial Distribution in Raw Milk. Food ence, 2018.39(16):186-191

4. Soto del Rio M, Dalmasso A, Civera T, Botter MT. Characterization of bacterial communities of donkey milk by high-throughput sequencing. International Journal of Food Microbiology, 2017.251: 67-72.

5. Gao JY, Shao YY, Lei FY, Wang BN, Zhang FX. Analysis of bacterial diversity in goats' milk from Guanzhong region of Shaanxi province. Food \& Fermentation Industries, 2018.44(01):52-59

6. Kongo M. Lactic Acid Bacteria - R \& D for Food, Health and Livestock Purposes. 2013.

7. Karimi R, Sohrabvandi S, Mortazavian AM. Review Article: Sensory Characteristics of Probiotic Cheese. Comprehensive Reviews in Food Science E Food Safety, 2012.11(5): 437-452.

8. Hansen A, Schieberle P. Generation of aroma compounds during sourdough fermentation: applied and fundamental aspects. Trends in Food Science \& Technology, 2005.16(1-3):85-94.

9. Pisano MB, Scano P, Murgia A, Cosentino S, Caboni P. Metabolomics and microbiological profile of Italian mozzarella cheese produced with buffalo and cow milk. Food Chemistry, 2016. 192: 618-624.

10. Ojala T, Laine PKS, Ahlroos T, Tanskanen J, Auvinen P. Functional genomics provides insights into the role of Propionibacterium freudenreichii ssp. shermanii JS in cheese ripening. International Journal of Food Microbiology, 2017.241:39-48

11. Jany JL, Barbier G. Culture-independent methods for identifying microbial communities in cheese. Food Microbiology, 2008. 25(7): 839-848.

12. Alegría A, Szczesny P, Mayo B, Bardowski J, Kowalczyk M. Biodiversity in Oscypek, a Traditional Polish Cheese, Determined by Culture-Dependent and -Independent Approaches. Appl Environ Microbiol, 2012.78(6):1890-1898

13. Vaz-Moreira I, Egas C, Nunea OC, Manaia CM. Bacterial diversity from the source to the tap: a comparative study based on 16S rRNA gene-DGGE and culture-dependent methods. Fems Microbiology Ecology, 2013.83(2):361-374

14. Shu C, Wu CS, Zhong CP, Suo HY, Li L. Progress of Research Methods for Microbial Diversity in Fermented Foods. Food ence. 2013.34(15): 397-402.

15. Riesenfeld CS, Schloss PD, Handelsman J. Metagenomics: genomic analysis of microbial communities. Annu.rev.genet, 2004.38(1): 525-552.

16. Qin JJ, Li RQ, Raes J, Arumugam M, Burgdorf K, Manichanh C, et al. A human gut microbial gene catalogue established by metagenomic sequencing. Nature, 2010. 464: 59-65.

17. Staley C, Sadowsky MJ. Application of metagenomics to assess microbial communities in water and other environmental matrices. Journal of the Marine Biological Association of the Uk, 2016.96(1):121-129.

18. Zhang WX, Li J, Qian Y,Suo HY. Research Progress and Applications of Metagenomics in Food 
Science. Food ence, 2012. 33(5):309-314.

19. Escobar-Zepeda A, Sanchez-Flores A, BaruchM Q. Metagenomic analysis of a Mexican ripened cheese reveals a unique complex microbiota. Food Microbiology, 2016. 57(8):116-127.

20. Gao J, Gu FY, He J, Xiao JZ, Chen QH, Ruan H, et al. Metagenome analysis of bacterial diversity in Tibetan kefir grains. European Food Research \& Technology, 2013. 236(3): 549-556.

21. Nalbantoglu U, Cakar A, Dogan H, Abaci N, Ustek D, Sayood K, et al. Metagenomic analysis of the microbial community in kefir grains. Food Microbiology, 2014. 41(8): 42-51.

22. Ceugniez A, Taminiau B, Coucheney F, Jacques P, Delcenserie V, Daube G, et al. Fungal diversity of "Tomme d'Orchies" cheese during the ripening process as revealed by a metagenomic study. International Journal of Food Microbiology, 2017.258: .89-93.

23. Zhong Z, Hou QC, Kwok LY, Yu Z, Zheng Y, Sun,Z.H, et al. Bacterial microbiota compositions of naturally fermented milk are shaped by both geographic origin and sample type. Journal of Dairy Science, 2016. 99(10): 7832-7841.

24. Gao ML, Hou HM, Teng XX, Zhu YL, Hao HS, Zhang GL. Microbial diversity in raw milk and traditional fermented dairy products (Hurood cheese and Jueke) from Inner Mongolia, China. Genetics \& Molecular Research, 2017. 16(1).

25. Sun ZH, Liu WJ, Bao QH, Zhang JC, Hou QC, KwoK LY, et al. Investigation of bacterial and fungal diversity in tarag using high-throughput sequencing. Journal of Dairy Ence, 2014.97(10): 6085-6096.

26. Systematics CM, Bioresources DO, ,Microbiological YCIF. Diversity of lactic acid bacteria and yeasts in Airag and Tarag, traditional fermented milk products of Mongolia. Japanese Journal of Lactic Acid Bacteria,2011,22 (3) :153-161.

27. Xu HY, Liu WJ, Gansudu QM, Sun ZH, Zhang JC, Gu Z,et al. Assessment of the bacterial and fungal diversity in home-made yoghurts of Xinjiang, China by pyrosequencing. Journal of the Science of Food \& Agriculture, 2015.95(10):2007-2015

28. Tothi T, Abdumilik N, Zhu XY, Rahman N. Gastrointestinal Tolerance of Dominant Lactic Acid Bacteria from Traditional Fermented Milk in Sayram, Baicheng County, Xinjiang, A Longevity Town of China. Journal of Dairy Science E Technology, 2015,38(03):14-16.

29. Chen C, Li W, Dong MS. Inhibition Effect of Skim Milk Fermented with Dominant Strains Isolated from Sayram Ketteki Yoghurt on Angiotensin-Converting Enzyme(ACE). Journal of Dairy Science \& Technology, 2012,35(01):20-24.

30. Lei HW, Chen XH, Li W, Wang Y, Peng J, Li S, et al.Analysis of Main Flavor Components in Milk Fermented by Original Strains from Sayram Ketteki. Food ence, 2013. 34(20): 127-130.

31. Caporaso JG, Kuczynski J, Stombaugh J, Bittinger K, Bushman FD, Costello EK, et al.QIIME allows analysis of high-throughput community sequencing data. Nature Methods, 2010, 7(5):335-336.

32. Magoč T, Salzberg SL. FLASH: fast length adjustment of short reads to improve genome assemblies. Bioinformatics,2011, 27(21):2957-2963..

33. Edgar RC. Search and clustering orders of magnitude faster than BLAST. Bioinformatics, 2010.

34. DeSantis TZ, Hugenholtz P, Larsen N, Rojas M, Brodie EL, Keller K, et al. Greengenes, a Chimera-Checked 16S rRNA Gene Database and Workbench Compatible with ARB. Applied $\mathcal{E}$ Environmental Microbiology. 2006, 72(7):5069-5072.

35. Abarenkov K, Nilsson RH, Larsson K, Alexande IJ, Eberhardt U, Erland S, et al. The UNITE database for molecular identification of fungi--recent updates and future perspectives. New 
Phytologist, 2010. 186(2):281-285.

36. Asnicar F, Weingart G, Tickle TL, Huttenhowe C, Segata N. Compact graphical representation of phylogenetic data and metadata with GraPhlAn. Peerj, 2015. 3(1):1029.

37. Langille MGI, Zaneveld J, Caporaso JG, Mcdonald D, Knights D, Reyes JA, et al. Predictive functional profiling of microbial communities using $16 \mathrm{~S}$ rRNA marker gene sequences. Nature Biotechnology, 2013. 31:814-821

38. Quigley L, O'Sullivan O, Beresford TP, Ross RP, Fitzgerald GF, Cotter PD. Molecular approaches to analysing the microbial composition of raw milk and raw milk cheese. International Journal of Food Microbiology, 2011, 150(2-3):81-94.

39. Chen ZL, Yang JX, Li MH, Li XW, Nan ZQ, Gong WF. Biodiversity and Quantitative Analysis of Lactic Acid Bacteria in Traditionally Fermented Milk Products from Seven Pasturing Areas of Tibet. Food ence, 2013. 34(17):140-145.

40. Liu WJ, Xi XX, Sudu QMG, Kwok LY, Guo Z, Hou,QC, et al.High-throughput sequencing reveals microbial community diversity of Tibetan naturally fermented yak milk. Annals of Microbiology, 2015. 65(3):1741-1751.

41. Han XY, Sun DQ, Li X, Huo GC, Jiang YJ. Gene regulation to lactic acid bacteria for increasing production of flavor metabolite. Acta Microbiologica Sinica, 2007, 47(6):1105-1109. ,

42. Dan T, Zhang HP. The Research Progress on Flavor Compounds in Fermented Milk. Journal of Chinese Institute of Food ence and Technology, 2018.18(11):287-292.

43. Han BZ, Meng Y, Min L, Yang YX, Ren FZ, Zeng QK, et al. A survey on the microbiological and chemical composition of buffalo milk in China. Food Control, 2007. 18(6): 742-746.

44. Quigley L, McCarthy R, O'Sullivan O, Beresford TP, Fitzgerald GF, Ross RP, et al. The microbial content of raw and pasteurized cow milk as determined by molecular approaches. Journal of Dairy Science, 2013,96(8):4928-4937

45. Zhang M, Zhang Y, Huang LL, Liu YD, Zhou H, Ni YQ. Application of 16S rDNA High-Throughput Sequencing for Comparative Study of the Microbial Diversity of Dairy Products from Western and Northern Xinjiang, China. Food Ence, 2017.,38(20):27-33

46. Ahat M, Ablikim X, Rahman N. Diversity of Culturable Microorganisms in Traditional Fermented Milk from South Xinjiang as Analyzed by High-Throughput Pyrosequencing. Food ence, 2018,39(20):126-131

47. Yoon WH, Nam BR, Kim JM, Kim CH. Characteristics of Functional Fermented Milk by Mixed Starters of Lactobacillus bulgaricus and Kluyveromyces marxianus. Hangug chugsan sigpum haghoeji = Korean journal for food science of animal resources, 2006. 26(2).

48. Zhang DD, Liu JL, Jiang TM, Li L, Fang GZ, Liu YP, et al. Influence of Kluyveromyces marxianus on proteins, peptides, and amino acids in Lactobacillus-fermented milk. Food ence and Biotechnology, 2017. 26(3):1-10. 
A

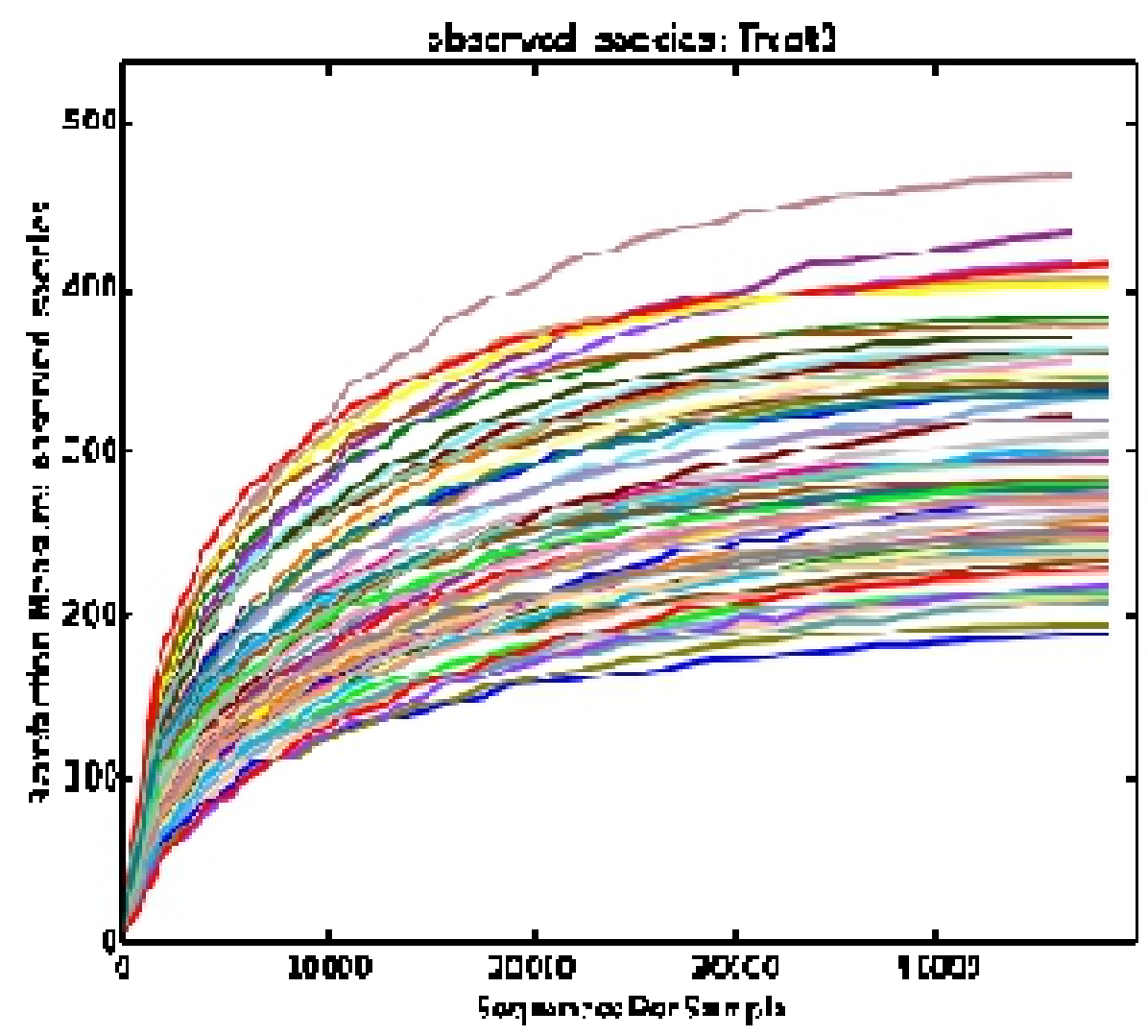

C

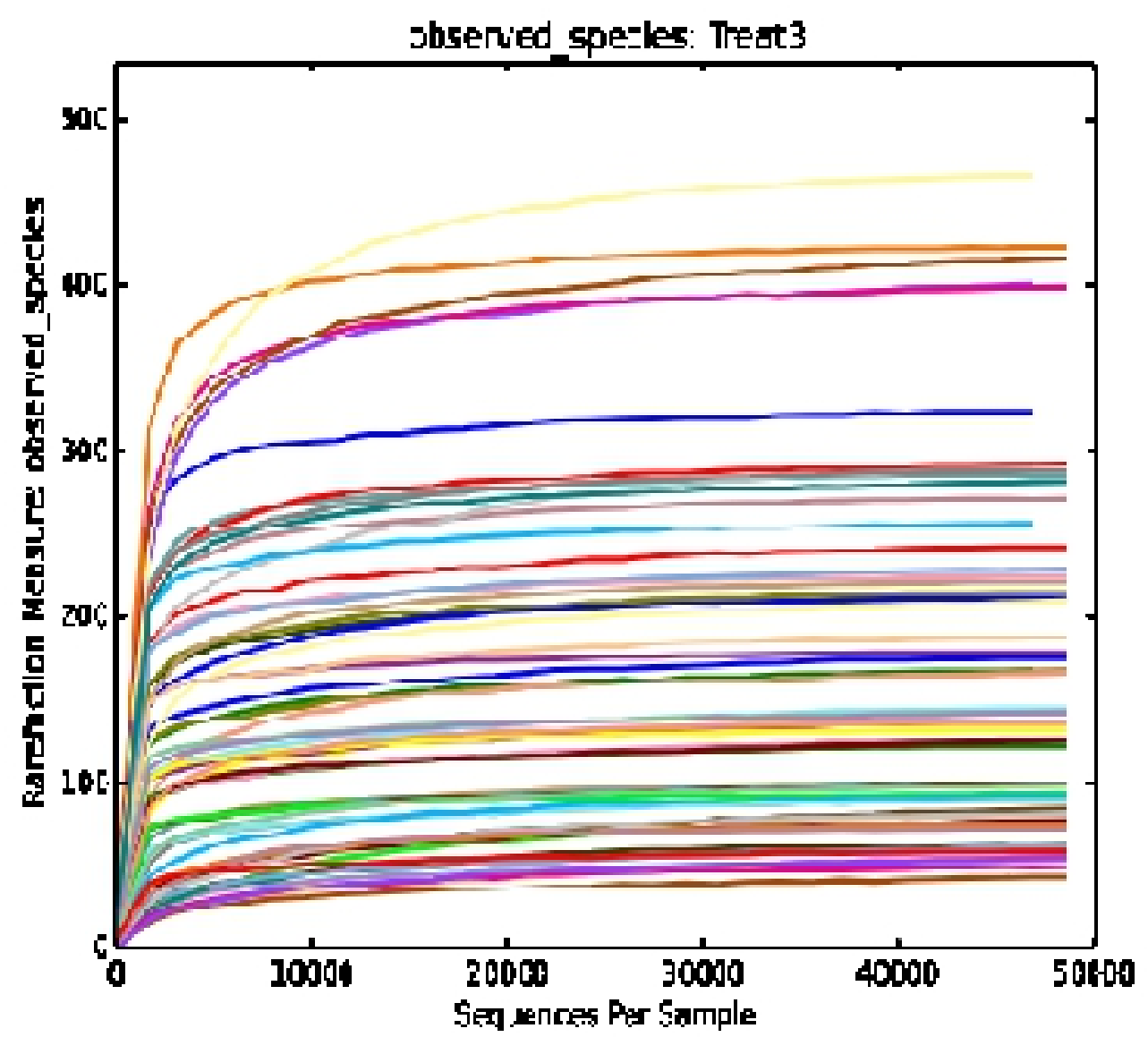

B

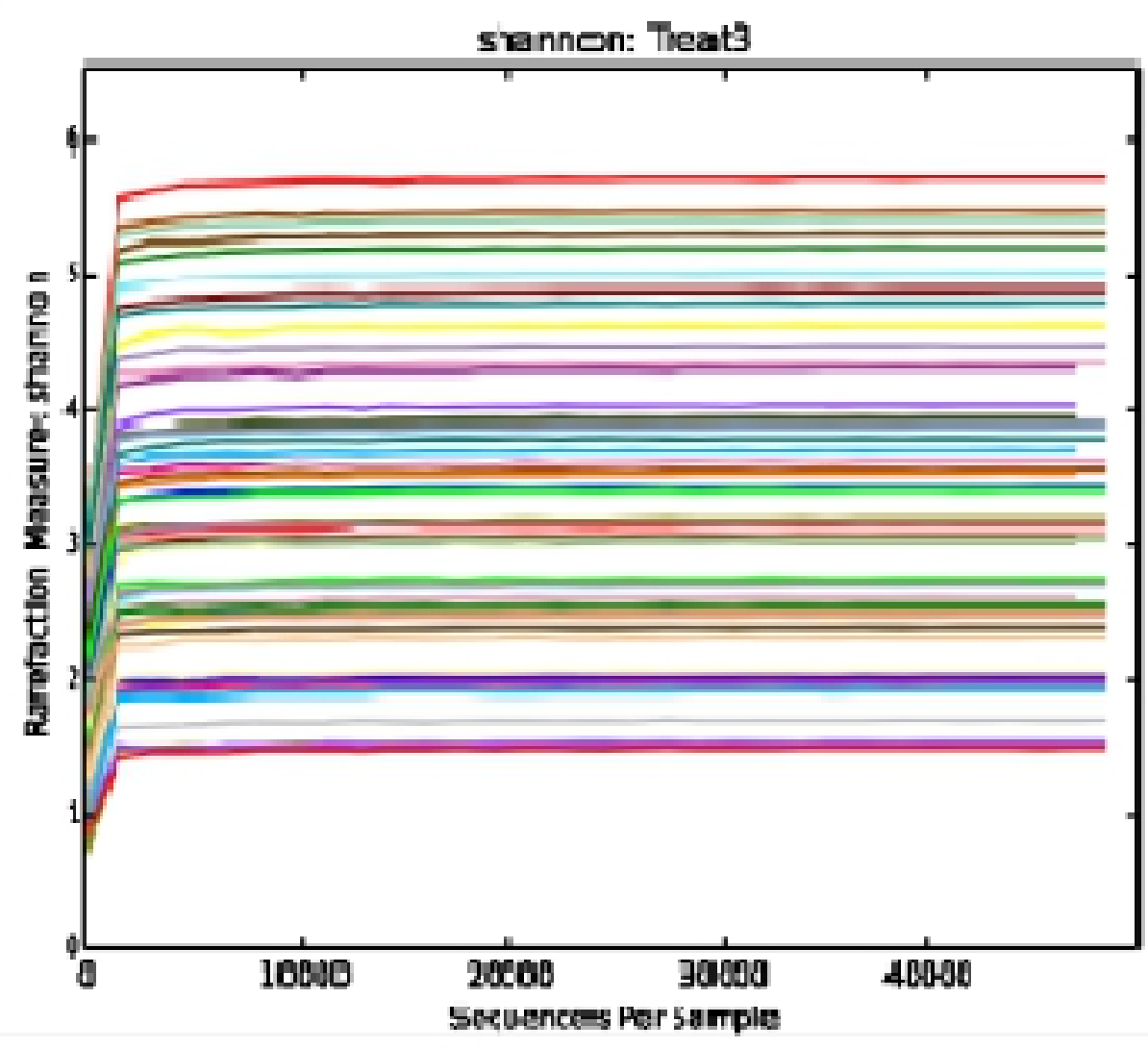

Bacterial rarefaction $(A)$, Shannon diversity $(B)$ and funga 
$\mathbf{A}$

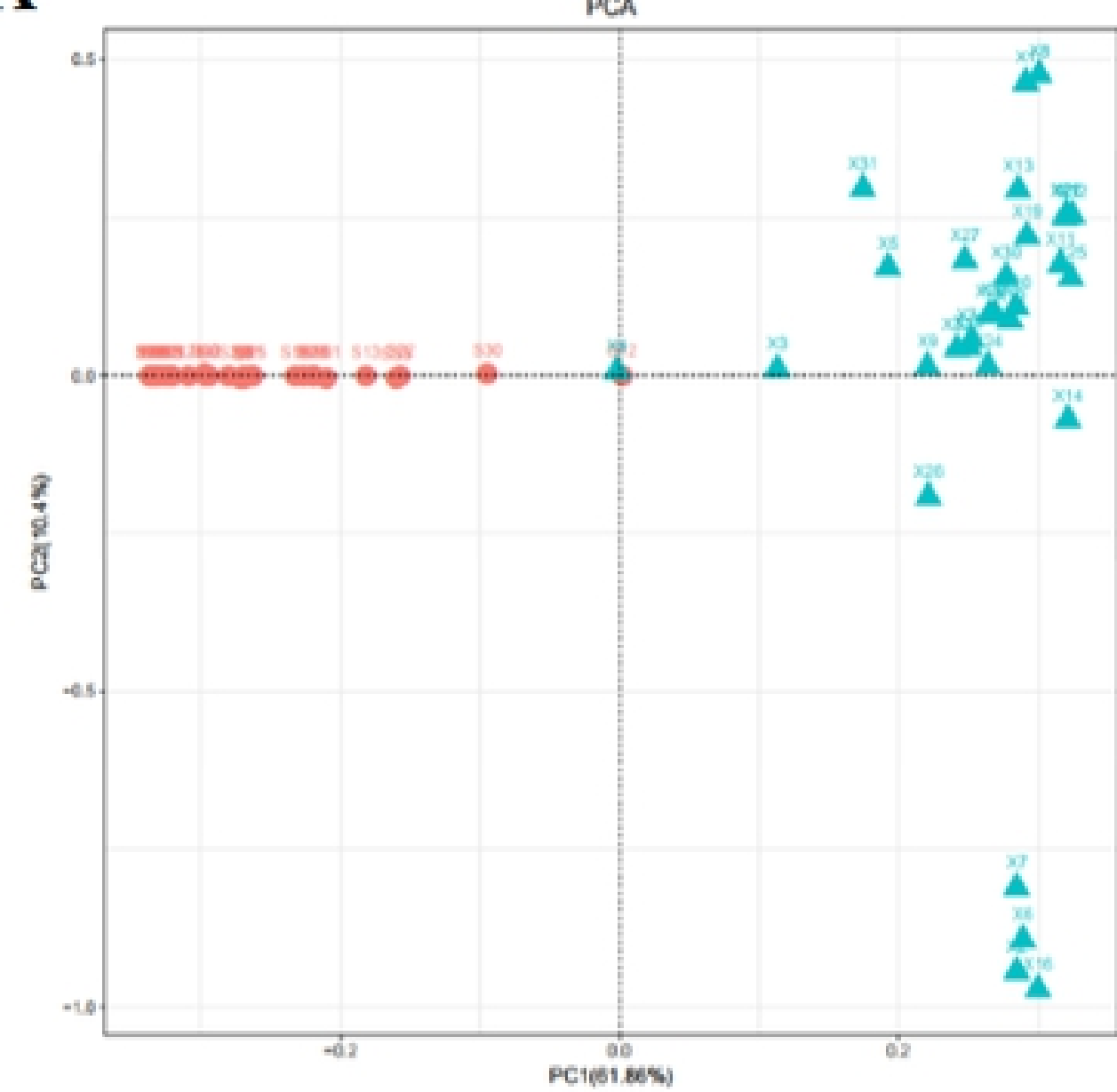

B

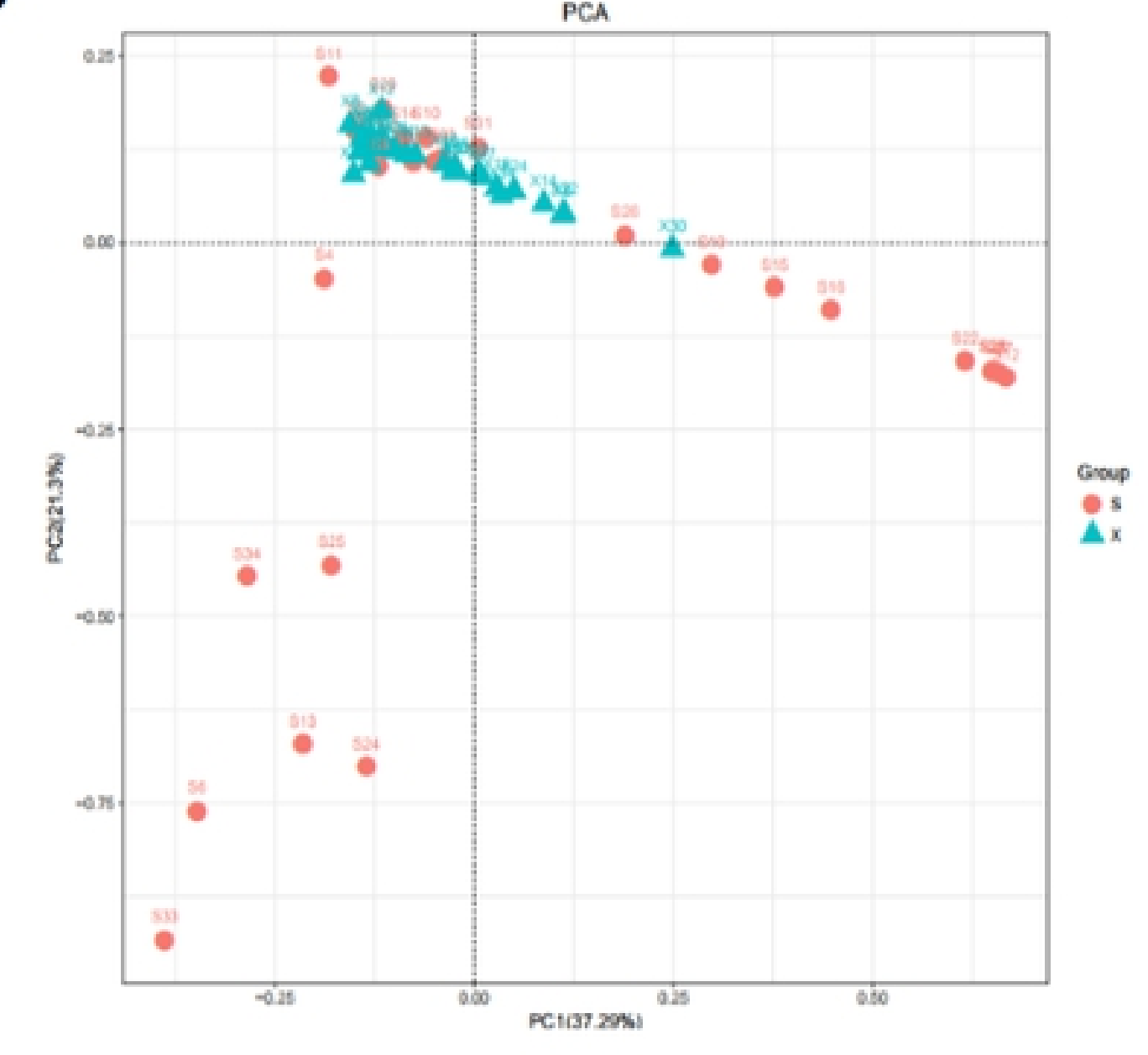

Principal component analysis (PCA) of bacterial (A) and fungal (B 


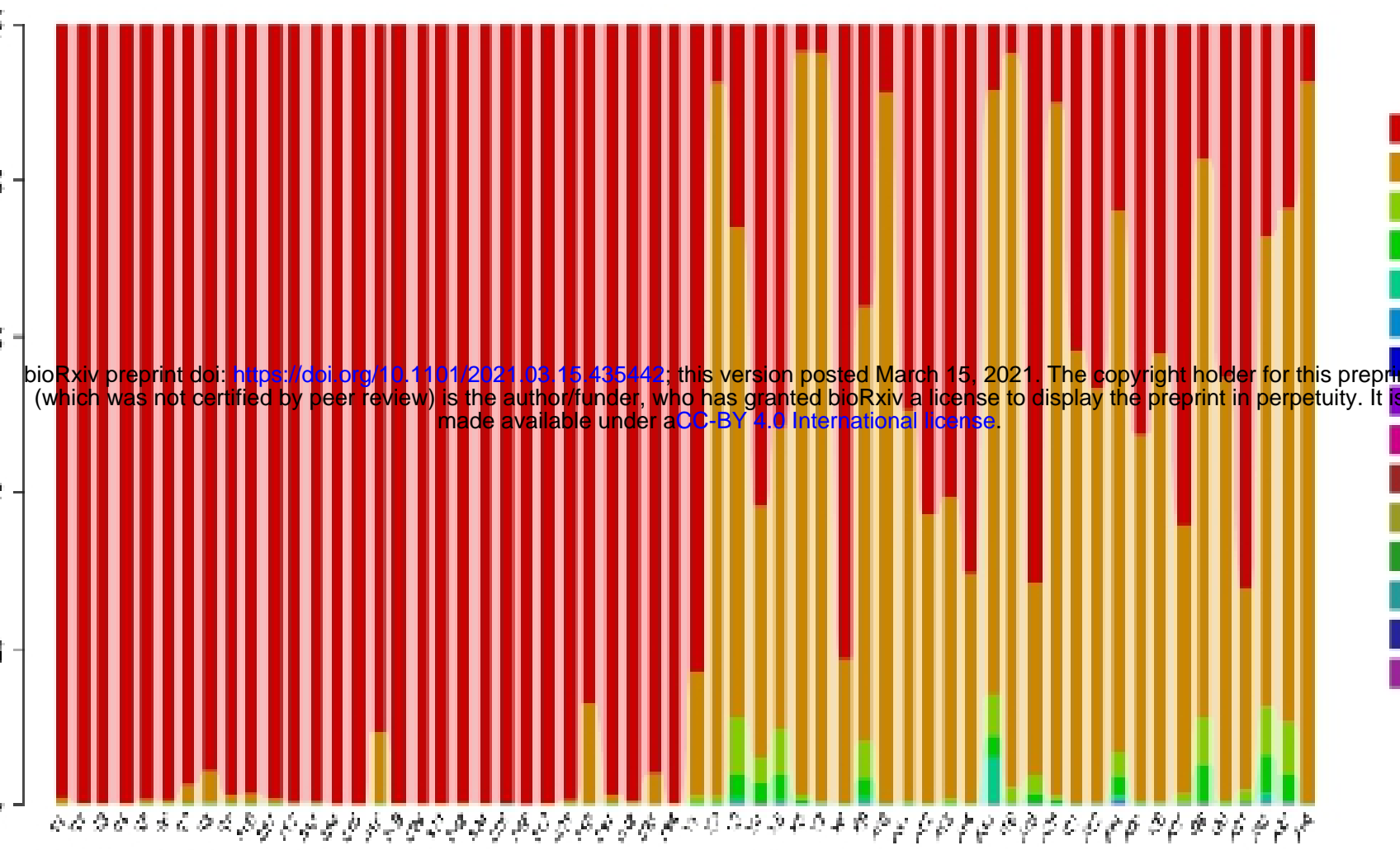

Firmicutes

Proteobacteria

Deinococcus-Thermus

Bacteroidetes

Actinobacteria

Cyanobacteria

Planctomycetes

Fusobacteria

Acidobacteria

Patescibacteria

Armatimonadetes

Verrucomicrobia

WPS-2

Elusimicrobia

Epsilonbacteraeota

B

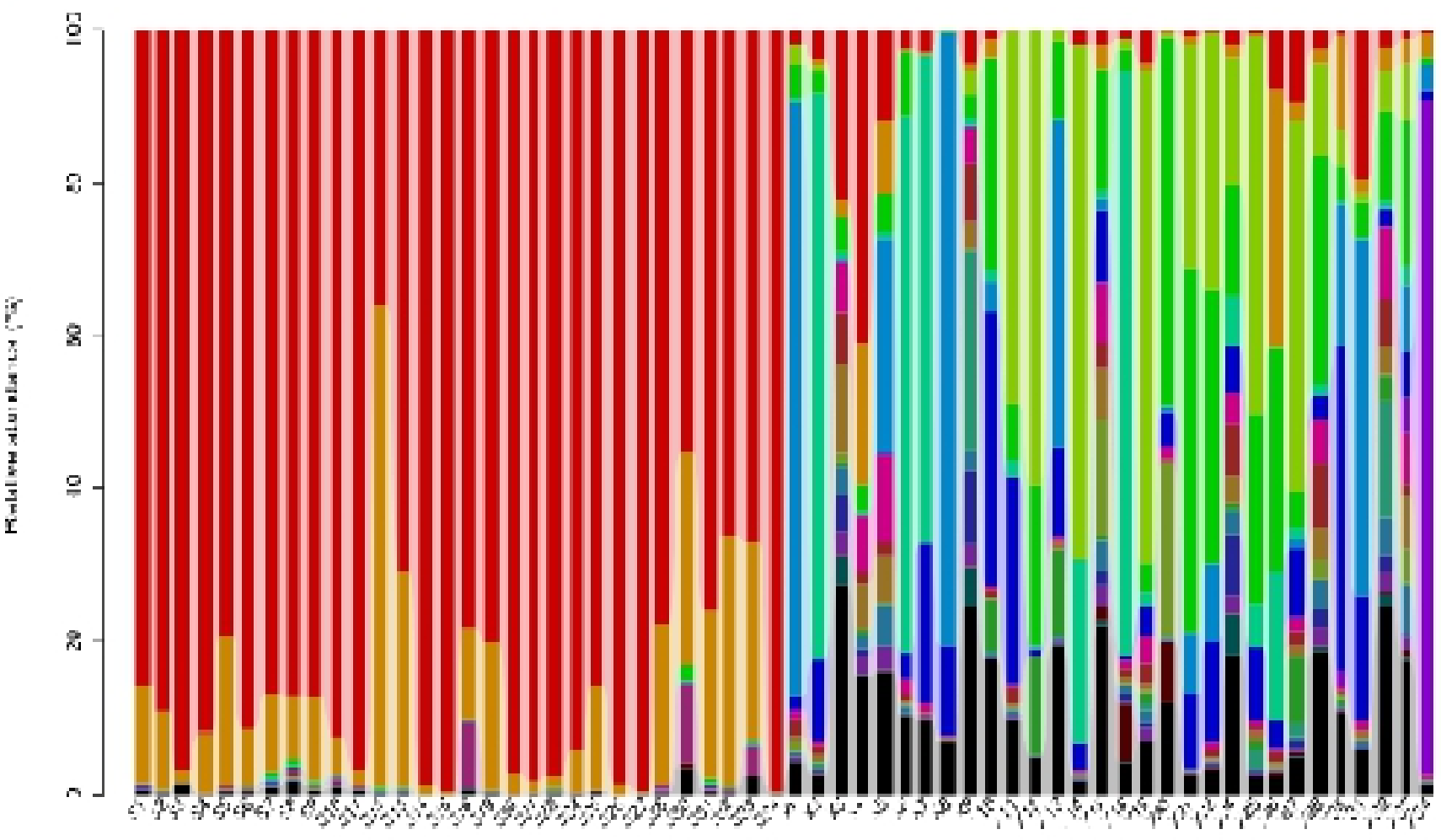

- Lactobacilus

Streotococcus

Macrococius

Acinetobacter

Iseudomanas

- Lactococous

- enterobacier

Kletsiella

- Ochrobacrum

Brevundimonas

- Ralstonic

Enh/drobacter

- Escherichia-Shigella

- Staphvioojccus

- Therrme

Dechlororronas

- Allorhizob um-Neorhizobium-Pararhizojiurr-Rhizobium

Acelobacter

- Aeromones

M tsuaria

- Others

C

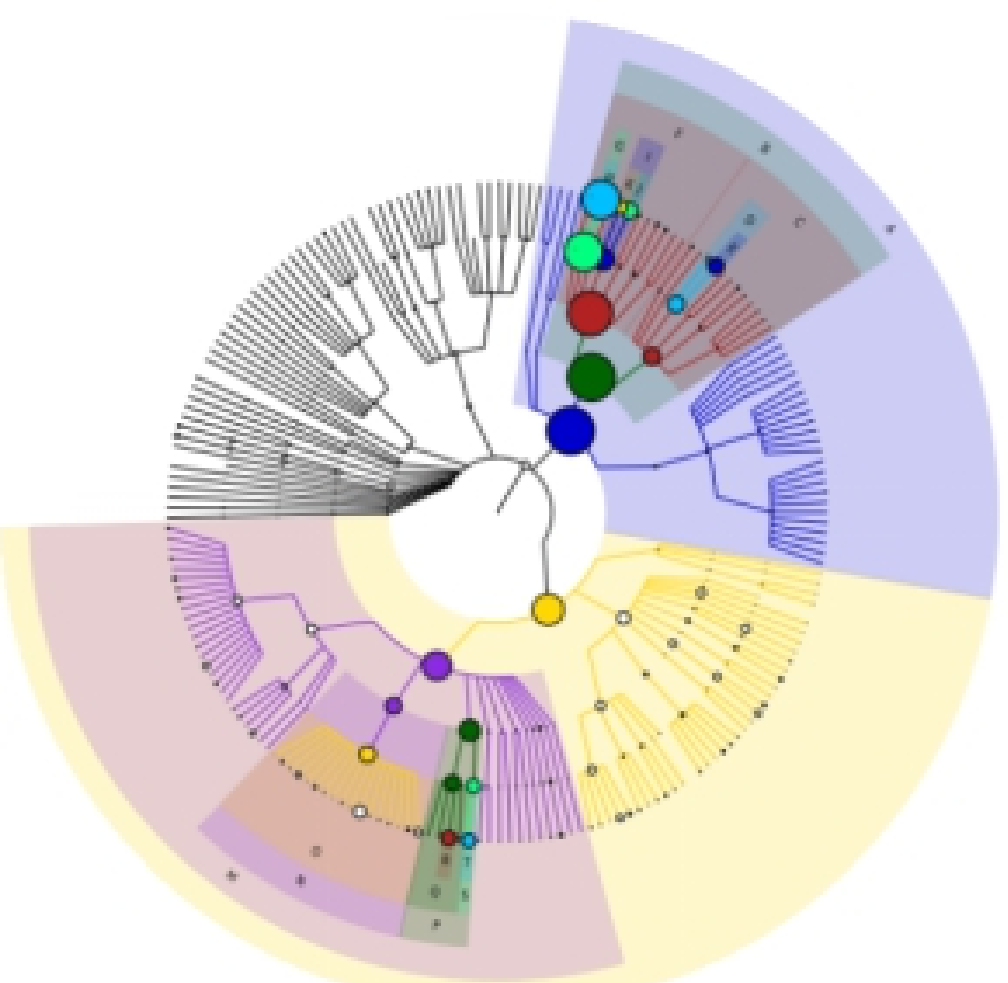

A:p Firmicutes

B:C_Bacilli

C:O-Bacillales

D:f Staphylococcaceae

E:g_Macrococcus

F:o Lactobacillales

G:f_Lactobacillaceae

$\mathrm{H}: \mathrm{g}$ - Lactobacillus

I:f Streptococcaceae

I: - Streptococcus

$\mathrm{K}: \mathrm{g}$ Lactococcus

L:p Proteobacteria

M:C Gammaproteobacteria

$\mathrm{N}: \mathrm{O}$ Enterobacteriales

O:f Enterobacteriaceae

P:o-Pseudomonadales

Q:f_Moraxellaceae

R:g Acinetobacter

S:f-Pseudomonadaceae

T:g Pseudomonas

\section{Relative abundances of bacteria at phylum (A) and genera}




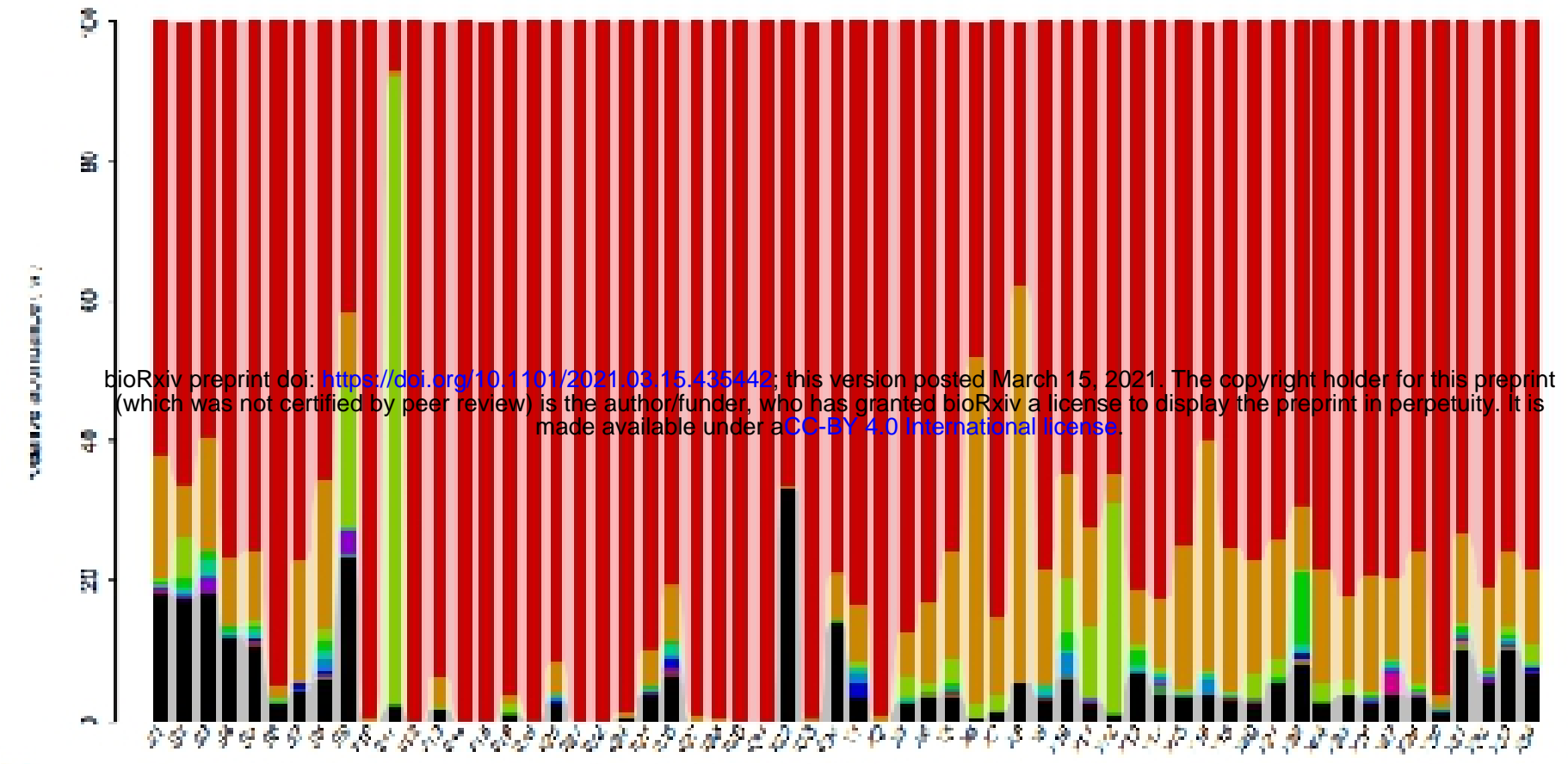

B

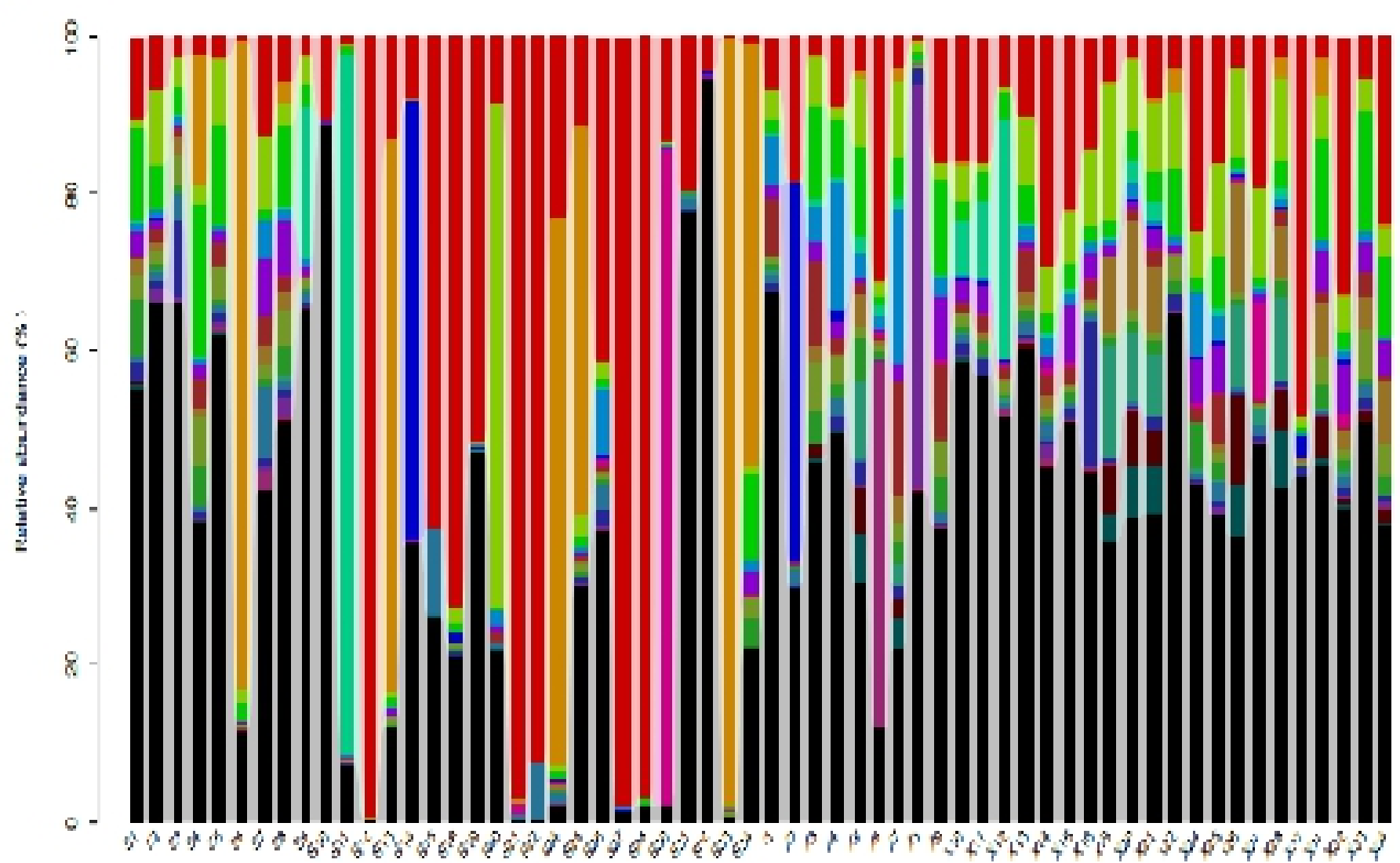

C

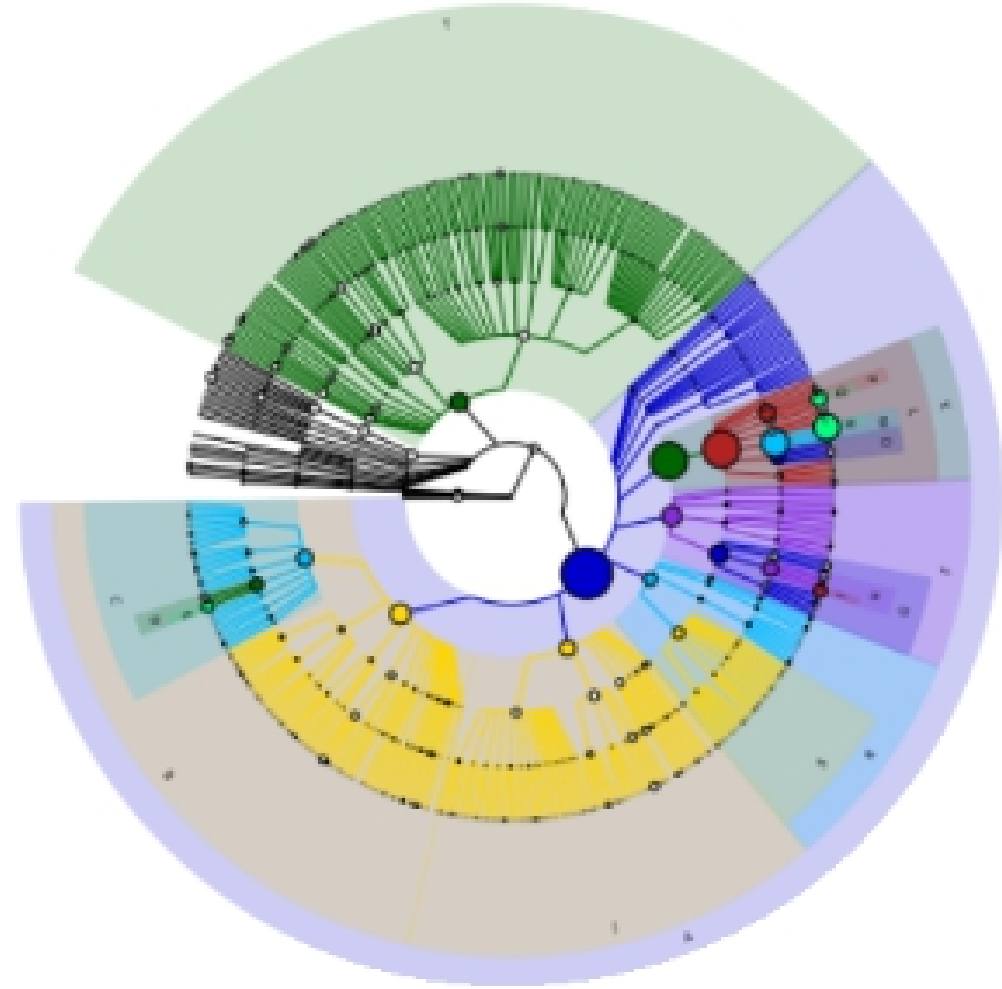

Ascomycota

Basidiomycota

- Mucoromycota

- Mortierellomycota

Rnzellomyrota

Olpidiunrycula

Anthophyte

- Entomophthoromycota

Cnidarla

Chytridiomycota

Cercozoa

- Ginmeromycota

Zoopagomycota

G319

- Chloroohyta

Ciliophora

Rutifera

Othere

\section{Candida \\ Kluyveromyces \\ Aspergillus \\ Lecanicillium \\ Mucor \\ Penicillium \\ Clavispora \\ Malassezia \\ Yarrowia \\ Cladosporium \\ Thermoascus \\ Simplicillium \\ Verticillium \\ Suillus \\ Pichia \\ Cutaneotrichosporon \\ Trichosporon \\ Rhodotorula \\ Thermomyces \\ Wickerhamomyces \\ Others}

A:p_Ascomycota

B:c Sordariomycetes

C:o_Hypocreales

D:f Cordycipitaceae

$\mathrm{E}: \mathrm{g}$ Lecanicillium

F:c_Eurotiomycetes

G:o_Eurotiales

H:f_Aspergillaceae

I:g Aspergillus

J:c Dothideomycetes

K:c Saccharomycetes

L:o-Saccharomycetales

M:f__Saccharomycetales_fam_Incertae_sedis

$\mathrm{N}: \mathrm{g}$ Candida

O:f Dipodascaceae

P:f_-Saccharomycetaceae

$\mathrm{Q}: \bar{g}$ Kluyveromyces

R:c Leotiomycetes

S:o Helotiales

T:p_Basidiomycota

\section{Relative abundances of fungi at phylum (A) and genera}


Mascococos
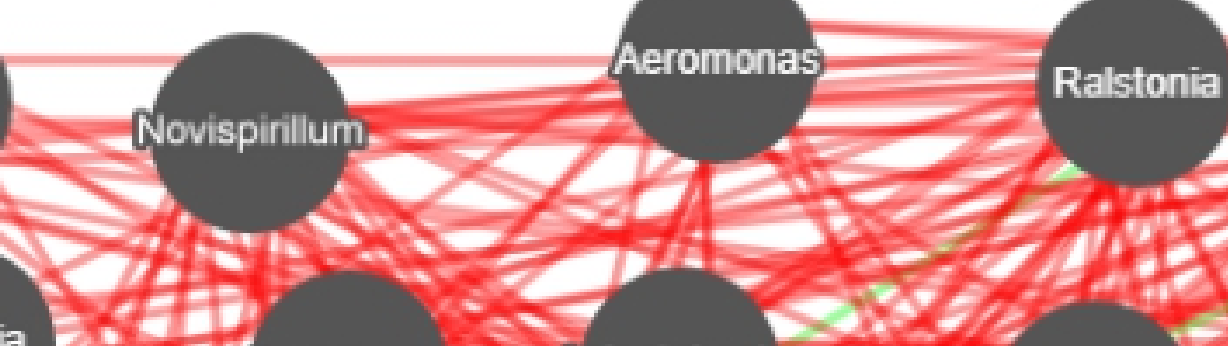

Caulobacter Acinetobacter 2 .

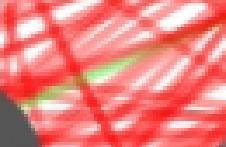

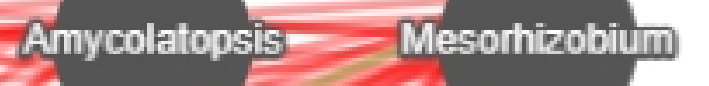

Brevundimonas Serratia

(1)

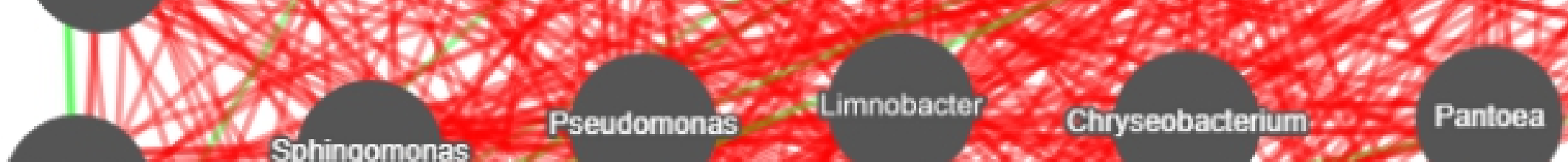
Methylobacterium-

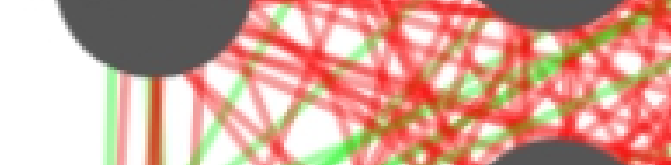
s.t.

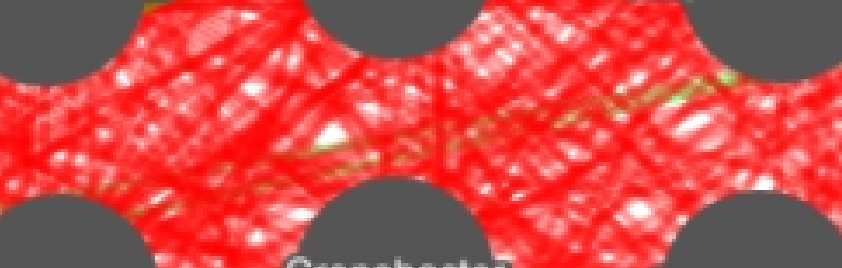
$3509=$ Pantoea Staphylococcus Lactobacillus

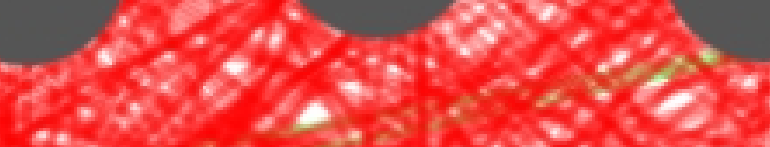
ose 20

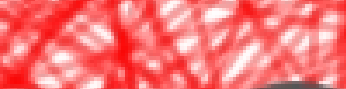
yesisy Vibrio

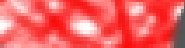
Delitia of Exiguobacterium $\cos _{3}$

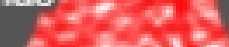
$800 \times 3$

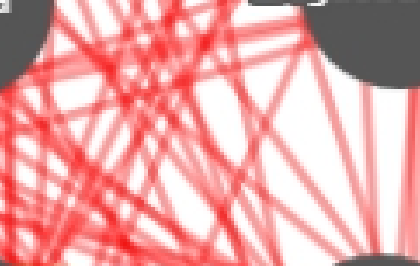
exwas on - .

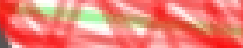
atilis Clo Enterobacter Paucibacter chloromonas 20.8

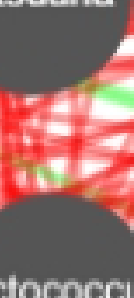
2 Wicrobacteriun Enhydrobacter
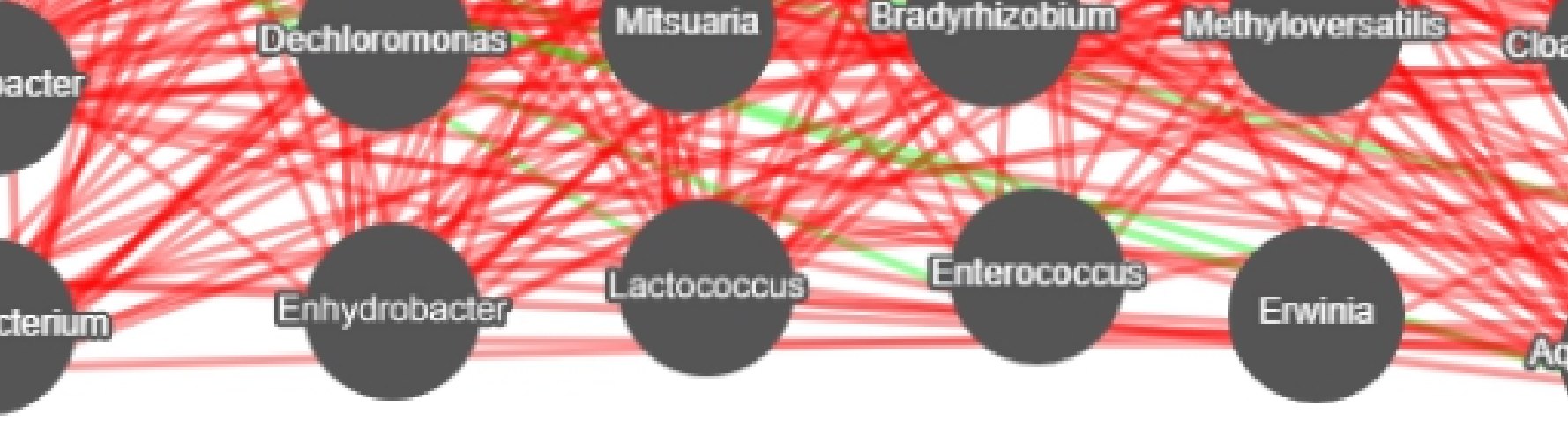
$2 \times 1$
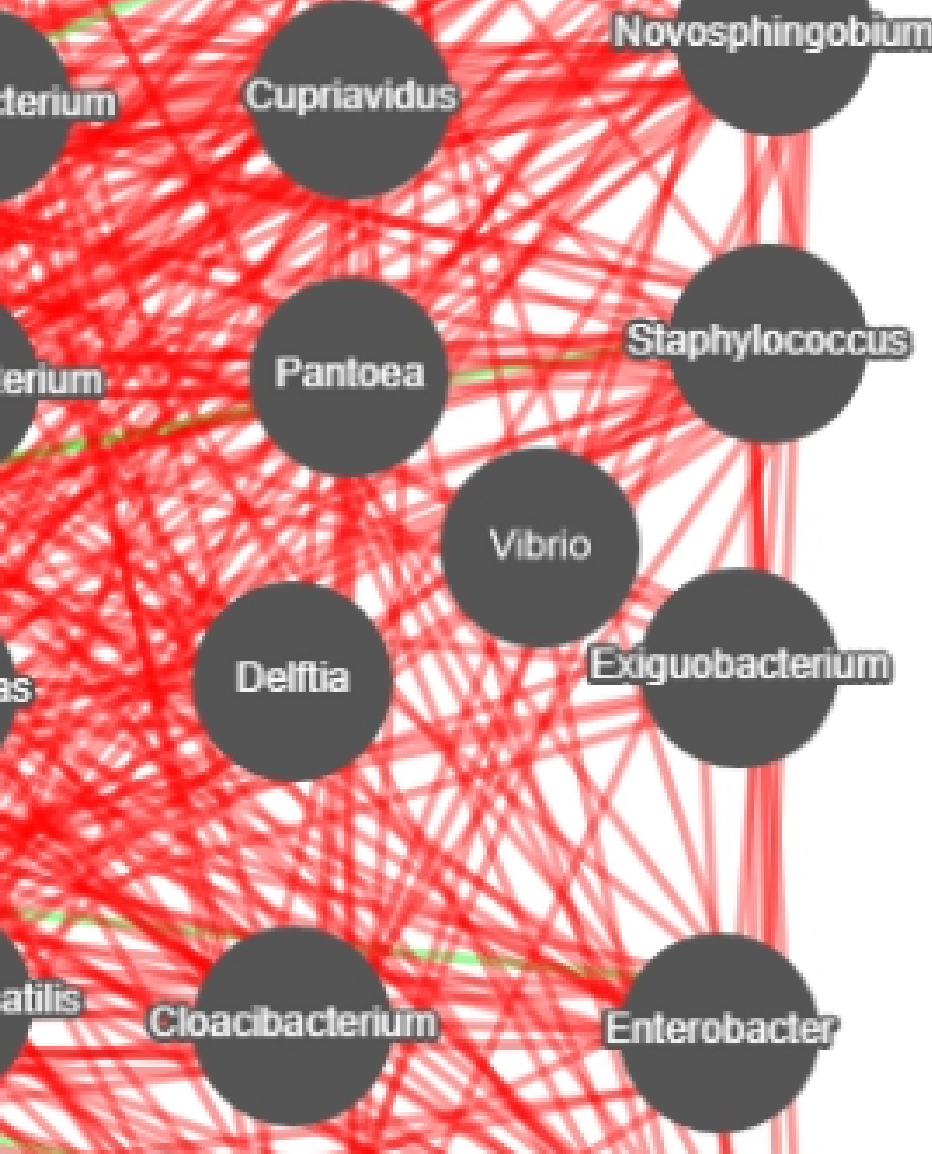

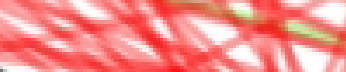

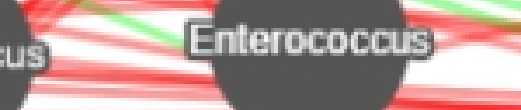

Erwinia

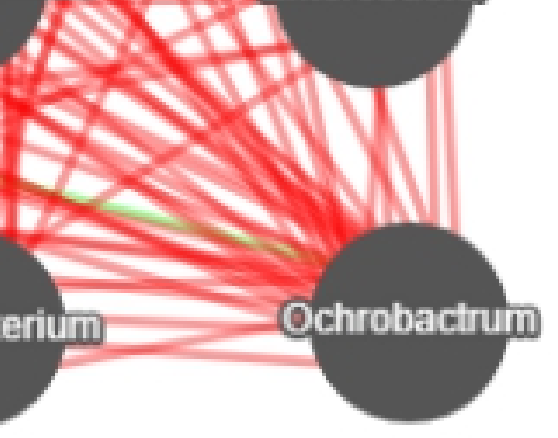

Dominant of top 50 bacterial genera association netwo 
Table 1: Alpha diversity values in samples

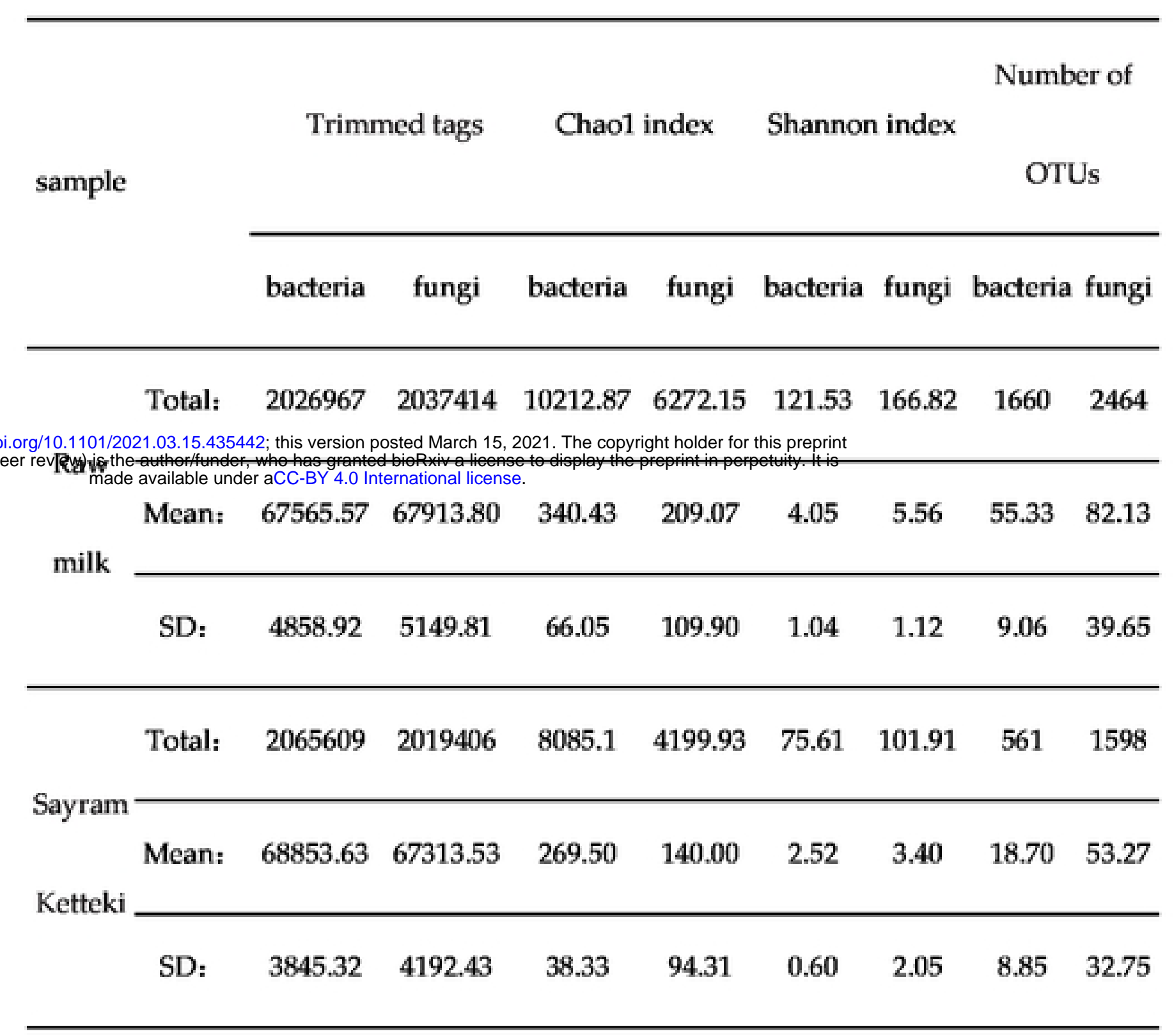

bioRxiv preprint doi: https://doi.org/10.1101/2021.03.15.435442; this version posted March 15, 2021. The copyright holder for this preprint (which was not certified by peer reviews) 
Table 2. Analysis of OTU Percentage of the top 20 genera

\begin{tabular}{|c|c|c|c|c|c|}
\hline \multirow{2}{*}{ Genera of Bacteria } & \multicolumn{2}{|c|}{ OTU Percentage (\%) } & \multirow{2}{*}{ Genera of Fungi } & \multicolumn{2}{|c|}{ OTU Percentage $(\%)$} \\
\hline & RM & SK & & RM & SK \\
\hline Lactobacillus & $5.1067 \pm 8.727$ & $85.5967 \pm 14.883$ & Candida & $13.400 \pm 11.517$ & $28.9233 \pm 35.865$ \\
\hline Streptococcus & $3.5533 \pm 7.047$ & $12.4667 \pm 13.646$ & Kluyveromyces & $0.4833 \pm 1.007$ & $14.8000 \pm 29.498$ \\
\hline Macrococcus & $15.3033 \pm 22.684$ & $0.0033 \pm 0.018$ & Aspergillus & $6.2967 \pm 4.311$ & $3.9433 \pm 11.780$ \\
\hline Acinetobacter & $13.1400 \pm 13.859$ & $0.2033 \pm 0.365$ & Lecanicillium & $4.9467 \pm 4.301$ & $2.9133 \pm 4.909$ \\
\hline 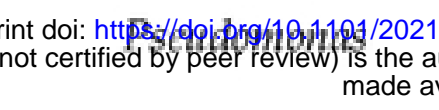 & 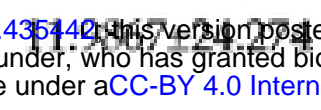 & 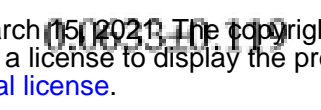 & $\begin{array}{l}\text { tholder for this preprint } \\
\text { print in perpeturty. It is }\end{array}$ & $2.1267 \pm 5.799$ & $3.6867 \pm 16.573$ \\
\hline Lactococcus & $11.1467 \pm 22.138$ & $0.0200 \pm 0.109$ & Penicillium & $2.9167 \pm 4.584$ & $0.8433 \pm 1.692$ \\
\hline Enterobacter & $8.9133 \pm 10.551$ & $0.0567 \pm 0.122$ & Clavispora & $1.7633 \pm 8.781$ & $1.9900 \pm 10.336$ \\
\hline Klebsiella & $3.2467 \pm 16.039$ & $0.0067 \pm 0.0365$ & Malassezia & $2.6133 \pm 2.385$ & $1.0200 \pm 1.941$ \\
\hline Ochrobactrum & $2.7500 \pm 3.114$ & $0.0533 \pm 0.073$ & Yarrowia & $0.6500 \pm 2.244$ & $2.9000 \pm 15.188$ \\
\hline Brevundimonas & $2.0067 \pm 2.398$ & $0.0933 \pm 0.151$ & Cladosporium & $2.7967 \pm 3.484$ & $0.6533 \pm 1.147$ \\
\hline Ralstonia & $2.0433 \pm 2.861$ & $0.0367 \pm 0.096$ & Thermoascus & $2.9467 \pm 3.831$ & $0.4533 \pm 0.807$ \\
\hline Enhydrobacter & $1.7067 \pm 4.902$ & $0.0067 \pm 0.025$ & Simplicillium & $1.8167 \pm 1.786$ & $1.1433 \pm 1.679$ \\
\hline Escherichia & $1.6700 \pm 3.396$ & $0.0067 \pm 0.025$ & Verticillium & $1.7100 \pm 1.453$ & $0.9067 \pm 1.689$ \\
\hline Staphylococcus & $1.6633 \pm 5.496$ & $0.0000 \pm 0.000$ & Suillus & $2.3433 \pm 4.163$ & $0.0267 \pm 0.146$ \\
\hline Thermus & $1.4267 \pm 1.737$ & $0.0367 \pm 0.056$ & Pichia & $0.8700 \pm 0.763$ & $1.4600 \pm 2.719$ \\
\hline Dechloromonas & $1.2000 \pm 2.364$ & $0.0033 \pm 0.018$ & Cutaneotrichosporon & $1.5433 \pm 3.325$ & $0.7800 \pm 1.825$ \\
\hline Allorhizobium & $0.9933 \pm 1.145$ & $0.0100 \pm 0.031$ & Trichosporon & $1.8533 \pm 9.383$ & $0.2067 \pm 0.549$ \\
\hline Acetobacter & $0.0167 \pm 0.046$ & $0.0073 \pm 0.024$ & Rhodotorula & $1.8100 \pm 8.485$ & $0.1500 \pm 0.395$ \\
\hline Aeromonas & $0.7133 \pm 1.933$ & $0.0002 \pm 0.001$ & Thermomyces & $1.8067 \pm 2.833$ & $0.0467 \pm 0.104$ \\
\hline Mitsuaria & $0.6733 \pm 1.441$ & $0.0001 \pm 0.001$ & Wickerhamomyces & $1.4667 \pm 2.552$ & $0.0700 \pm 0.195$ \\
\hline
\end{tabular}

